\title{
Model Based Nonlinear Iterative Learning Control: A Constrained Gauss-Newton Approach
}

\author{
M. Volckaert, A. Van Mulders, J. Schoukens, M. Diehl, J. Swevers
}

\begin{abstract}
A new method is proposed to solve the model inversion problem that is part of model based iterative learning control (ILC) for nonlinear systems. The model inversion problem consists of finding the input signal corresponding to a given output signal. This problem is formulated as a nonlinear dynamic optimization problem in time domain and solved efficiently using a constrained Gauss-Newton algorithm. A nonlinear ILC algorithm based on this model inversion approach is validated numerically and experimentally. The considered application is an electric circuit described by a polynomial nonlinear state-space model. The nonlinear ILC algorithm shows fast convergence and accurate tracking control.
\end{abstract}

Index Terms-ILC, Learning control systems, nonlinear systems, optimization methods

\section{INTRODUCTION}

The purpose of the research presented in this paper is to accurately control nonlinear systems, in other words to make nonlinear systems produce a predefined output. An often used approach to achieve this is feedback control. This approach has a number of well-known drawbacks: feedback introduces lag and stability issues. An alternative approach is feedforward control, which doesn't experience these drawbacks but which is not robust with respect to system variations and model inaccuracies. Iterative learning control (ILC) is an open loop control scheme that combines the benefits of feedback and feedforward control for repeated tasks by updating the control signal based on the performance during (the) previous repetition(s).

The research presented in this paper is a continuation of the work of Kris Smolders. He proposed an ILC law in [1] that provides accurate tracking control for nonlinear systems. The control law is an adaptation of the internal model control law, and makes use of the inverse of a nonlinear model. This model inverse is calculated by solving a dense optimization problem in the frequency domain, using a Newton algorithm. However, the current research focuses on the calculation of the model inverse by solving a sparse optimization problem in the time domain, using a constrained Gauss-Newton algorithm. This improves the efficiency of the

M. Volckaert and J. Swevers are with the Department of Mechanical Engineering, Katholieke Universiteit Leuven, Celestijnenlaan 300B, B-3001 Leuven, Belgium (Marnix.Volckaert@mech.kuleuven.be)

A. Van Mulders and J. Schoukens are with the Department ELEC, Vrije Universiteit Brussel, Pleinlaan 2, B-1050 Brussels, Belgium

M. Diehl is with the Department of Electrical Engineering, Katholieke Universiteit Leuven, Kasteelpark Arenberg 10, B-3001 Leuven, Belgium

This work benefits from FWO-project G.0422.08, K.U.Leuven-BOF EF/05/006 Center-of-Excellence Optimization in Engineering and the Belgian Programme on Interuniversity Attraction Poles, initiated by the Belgian Federal Science Policy Office (DYSCO). model inversion, which has to be performed during each iteration of the iterative learning control.

This paper is organised as follows: section II introduces both linear and nonlinear model based ILC. Section III presents the model inversion approach using the constrained Gauss-Newton algorithm. Section IV discusses the numerical and experimental validation of the proposed method, followed by conclusions in section $\mathrm{V}$.

\section{LINEAR AND NONLINEAR ITERATIVE LEARNING CONTROL}

Iterative learning control is an open loop control scheme that iteratively learns a feedforward signal based on the tracking error of (the) previous trial(s) in order to achieve accurate tracking [2]. Accurate tracking control is specified as to minimise the RMS value of the tracking error, that is:

$$
\min _{u}\left\|y_{r}-P(u)\right\|_{2}
$$

for a given system $P$, with $y_{r}$ being the reference signal, and $u$ the input.

A distinction can be made between linear and nonlinear ILC algorithms. Linear ILC uses a linear mapping of the tracking error to the input update, while nonlinear ILC uses a nonlinear mapping. Model based ILC methods make use of a model of the system for the mapping, so it is clear that model based linear ILC uses a linear model, while model based nonlinear ILC uses a nonlinear model.

The basic model based linear ILC law can be written as:

$$
u^{[i+1]}=u^{[i]}+Q^{[i]}\left[\hat{P}_{L}^{-1}\left(e^{[i]}\right)\right]
$$

with $e^{[i]}=\left[y_{r}-P\left(u^{[i]}\right)\right]$ the tracking error and $Q^{[i]}$ the iteration gain, at the iteration $[i] . \hat{P}_{L}$ is a linear model of the plant $P . \hat{P}_{L}$ can be either a parametric model, such as a state-space model, or a non parametric model, such as a measured impulse response or frequency response function (FRF).

The iteration gain is used to control the magnitude of the update to the input. It is common to initialise $Q$ as 1 , and to reduce its value when necessary [1]. One way to do this is to run the system with an updated input $u^{[i *]}$, calculated using a proposed value for $Q$, and accepting this value only if the newly measured output $y^{[i *]}$ shows a reduced tracking error. If an increase of the tracking error occurs, $Q$ is reduced and a new experiment is performed until the tracking error reduces. A successful update brings $Q$ back to its initial value. This approach is used in [1], and will be called approach 1 . Another approach is to always accept updated input signals, 


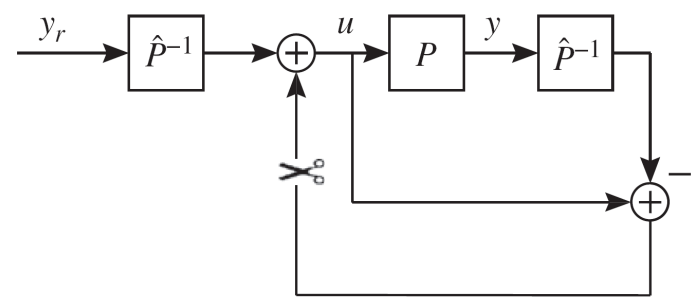

Fig. 1. Adapted nonlinear internal model control scheme

regardless of the tracking error, but to reduce $Q$ after an increase in tracking error without ever increasing or resetting $Q$ back to its original value. The idea behind this is that once accurate tracking is achieved, no update is needed on the input signal, hence $Q \approx 0$. This will be called approach 2.

Linear ILC algorithms are often not suitable for highly nonlinear systems. In the presence of strong nonlinearities, the linear ILC algorithm may converge slower, or fail to converge. Such systems can be accurately described by nonlinear models, so there is a need for model based nonlinear ILC. [1] introduces such an algorithm, as an adaptation of a nonlinear internal model control scheme, shown in figure 1. This scheme contains the following algebraic loop:

$$
u=u+\hat{P}_{N L}^{-1}\left(y_{r}\right)-\hat{P}_{N L}^{-1}(y) .
$$

By cutting the loop as indicated in figure 1, the loop is transformed into the following nonlinear iterative learning control law:

$$
u^{[i+1]}=u^{[i]}+Q^{[i]}\left[\hat{P}_{N L}^{-1}\left(y_{r}\right)-\hat{P}_{N L}^{-1}\left(y^{[i]}\right)\right] .
$$

In this equation $y^{[i]}=P\left(u^{[i]}\right)$ is the measured output at iteration $[i]$, and again the gain matrix $Q^{[i]}$ is added to control the size of the update at each iteration. $\hat{P}_{N L}$ is a nonlinear model of the plant $P$. The iterative control is initialised using the initial feedforward signal $u^{[0]}=\hat{P}_{N L}^{-1}\left(y_{r}\right)$.

The crucial step in the ILC law is the inversion of the model $\hat{P}_{N L}$, in other words the calculation of an input signal $u$ that produces a given output signal $y^{*}$. In [1] the inversion of the nonlinear model $\hat{P}_{N L}$ is performed by a Newton type optimization algorithm. The input signal is calculated iteratively using the inverse Jacobian of the model with respect to the input. The calculation of the inverse Jacobian is performed in the frequency domain. However, this method has a number of drawbacks: because the Jacobian is a dense matrix, the calculation of its inverse is computationally expensive, while this calculation has to be performed for every iteration of the Newton algorithm. Furthermore, the inversion can only be performed up to a maximal frequency, in order to limit the number of optimization variables. This means that the learning behaviour of an ILC law based on this inversion method is also limited to the same maximal frequency.

Section III presents a new method of solving the nonlinear model inversion problem in the time domain instead of the frequency domain, by formulating it as a nonlinear dynamic optimization problem. Both states and input are optimization variables, such that the problem has a favourable structure, which can be exploited by the algorithm. It is shown that this method does not experience the same drawbacks as the method presented in [1].

\section{MODEL INVERSION USING A CONSTRAINED GAUSS-NEWTON APPROACH}

The main problem to be solved in the proposed nonlinear ILC algorithm (3) is the nonlinear model inversion, that is finding an input signal $u$ corresponding to a given output signal $y^{*}$, for a nonlinear model $\hat{P}_{N L}$. From equation (3) it is clear that this inversion has to be performed once for the reference output $y_{r}$, and at each iteration $[i]$ for the measured output $y^{[i]}$. This problem is formulated here as a nonlinear dynamic optimization problem and solved efficiently using a constrained Gauss-Newton algorithm. The presented inversion method is valid for any type of discretetime nonlinear model, provided that the partial derivatives of the model equations with respect to the input and the states are known. For an extensive overview of nonlinear model types, such as black box models, block structured models, neural networks and fuzzy models, the reader is referred to [3]. In this section the model equations will be written as $x_{k+1}=f\left(x_{k}, u_{k}\right)$ and $y_{k}=h\left(x_{k}, u_{k}\right)$, with $f$ and $h$ nonlinear functions, and $k$ the time index.

\section{A. Model inversion formulated as a dynamic optimization problem}

The model equations $f$ and $h$ map the input $u$ to the output $y$ via the states $x$. For a given output $y^{*}$ the corresponding input $u$ can be found by solving the following dynamic optimization problem:

$$
\begin{aligned}
\min _{x, u} & \frac{1}{2}\left\|h\left(x_{k}, u_{k}\right)-y_{k}^{*}\right\|_{2} \\
& \text { s.t. } \\
f\left(x_{k}, u_{k}\right)-x_{k+1}=0 & \text { for } k=1,2, \ldots, N-1 \\
f\left(x_{N}, u_{N}\right)-x_{1}=0 &
\end{aligned}
$$

with $N$ the number of considered samples. Equation (4b) describes the system dynamics. For periodic reference trajectories with $N$ samples, equation (4c) ensures that the state at $k=N+1$ is equal to the state at $k=1$, and therefore guarantees periodicity of the solution. This can be a requirement in some applications, such as vibration test rigs [4]. Equation (4) is a nonlinear least squares problem. Such problems can be written in a general form as:

$$
\begin{gathered}
\min _{w} \frac{1}{2}\left\|F_{1}(w)\right\|_{2} \\
\text { s.t. } \\
F_{2}(w)=0 .
\end{gathered}
$$

In this case the variable vector consists of both the states $x$ and input $u$ at every considered time step, so $w=$ 
$\left[x_{1}, u_{1}, x_{2}, u_{2}, \ldots, x_{N}, u_{N}\right]^{T}$. The function $F_{1}(w)$ is a vector function of the so-called residuals, defined as:

$$
F_{1}(w)=\left[\begin{array}{c}
h\left(x_{1}, u_{1}\right)-y_{1}^{*} \\
h\left(x_{2}, u_{2}\right)-y_{2}^{*} \\
\vdots \\
h\left(x_{N}, u_{N}\right)-y_{N}^{*}
\end{array}\right]
$$

and $F_{2}(w)$ is a vector function of constraints, defined as:

$$
F_{2}(w)=\left[\begin{array}{c}
f\left(x_{1}, u_{1}\right)-x_{2} \\
f\left(x_{2}, u_{2}\right)-x_{3} \\
\vdots \\
f\left(x_{N}, u_{N}\right)-x_{1}
\end{array}\right] .
$$

Remark that the set of variables in the dynamic optimization problem (4) consists of the input and state variables. The relation between both, i.e. the system model dynamics, are described by the constraint equation (4b). Simultaneously optimizing on both inputs and states provides more degrees of freedom to the optimization algorithm and allows to initialize it at a guess for the states, which are often much better known than the inputs. This makes this simultaneous approach more robust compared to the conventional sequential approach to dynamic optimization, which eliminates the states for given inputs in each iteration. This in particular allows to much better treat unstable or even chaotic systems [5]. Problems of the form (5) can be solved efficiently by a constrained Gauss-Newton algorithm (CGN).

\section{B. Constrained Gauss-Newton algorithm for nonlinear least squares problems}

The constrained Gauss-Newton (CGN) algorithm is designed to solve nonlinear least squares problems of the form (5). Its convergence properties have been investigated, and widely been applied by Bock and coworkers [6], [7], [8]. The algorithm works iteratively, by making steps in a search direction that is found by the solution of a local, linear least squares problem. In this sense it is similar to a sequential quadratic programming algorithm, which sequentially calculates the search direction by solving linear quadratic problems [9].

The iterations over the variable vector $w$ can be written as $w^{i+1}=w^{i}+\alpha \Delta w$, with $\Delta w$ the search direction, and $\alpha$ the stepsize. As in most Newton type algorithms the stepsize is taken as 1 .

The search direction $\Delta w$ is determined by the solution of the linear least squares problem, that is found by linearizing equation (5) around the current iterate of the variable vector, so $w=w^{i}$. The linear sub-problem is:

$$
\begin{gathered}
\min _{\Delta w} \frac{1}{2}\left\|F_{1}(w)+J_{1}(w) \cdot \Delta w\right\|_{2} \\
\text { s.t. } \\
F_{2}(w)+J_{2}(w) \cdot \Delta w=0 .
\end{gathered}
$$

In this equation $J_{1}(w)$ and $J_{2}(w)$ are the Jacobians of the functions $F_{1}(w)$ and $F_{2}(w)$ respectively. For ease of notation the dependance of $F_{1}, F_{2}, J_{1}$ and $J_{2}$ on $w$ will be omitted. The optimal solution of the constrained linear least squares problem, called $\Delta w^{*}$, and the associated vector of Lagrange multipliers are obtained by solving the following set of linear equations:

$$
\underbrace{\left[\begin{array}{cc}
J_{1}^{T} J_{1} & -J_{2}^{T} \\
J_{2} & 0
\end{array}\right]}_{K K T}\left[\begin{array}{c}
\Delta w^{*} \\
\lambda^{*}
\end{array}\right]=\left[\begin{array}{c}
-J_{1}^{T} F_{1} \\
-F_{2}
\end{array}\right] .
$$

The matrix KKT is called the Karush-Kuhn-Tucker matrix. This equation uniquely defines $\Delta w^{*}$, which optimizes the linear least squares problem written in equation (8). $\Delta w^{*}$ is used as the optimal search direction to iterate the variable vector $w$.

Two criteria to stop the iterative search are commonly used. Closer to the optimum both the objective gradient and the constraint residuals will get closer to zero, and therefore the norm of $\Delta w$ will get smaller. The iterations can be stopped once $\Delta w$ has become sufficiently small. Another option is to use the norm of the right hand side of equation (9), which decreases as $w$ approaches its optimal value.

The advantage of the constrained Gauss-Newton algorithm for the model inversion problem, written in equation (4), lies in the structure of the Jacobian matrices $J_{1}$ and $J_{2}$. Using the definition of the functions $F_{1}$ and $F_{2}$, from (6) and (7), $J_{1}$ and $J_{2}$ are defined as:

$$
\begin{gathered}
J_{1}(w)=\left[\begin{array}{ccccc}
{\left[\frac{\partial h}{\partial x_{1}}\right]} & {\left[\frac{\partial h}{\partial u_{1}}\right]} & 0 & 0 & \cdots \\
0 & 0 & {\left[\frac{\partial h}{\partial x_{2}}\right]} & {\left[\frac{\partial h}{\partial u_{2}}\right]} & \cdots \\
\vdots & \vdots & \vdots & \vdots & \ddots
\end{array}\right] \\
J_{2}(w)=\left[\begin{array}{ccccc}
{\left[\frac{\partial f}{\partial x_{1}}\right]} & {\left[\frac{\partial f}{\partial u_{1}}\right]} & -I^{n \times n} & 0 & \cdots \\
0 & 0 & {\left[\frac{\partial f}{\partial x_{2}}\right]} & {\left[\frac{\partial f}{\partial u_{2}}\right]} & \cdots \\
\vdots & \vdots & \vdots & \vdots & \ddots \\
-I^{n \times n} & 0 & 0 & 0 & \cdots
\end{array}\right]
\end{gathered}
$$

It is clear that the structure of these matrices is sparse, which allows very efficient storage and manipulation in a computer algorithm. The matrices are also easy to construct, since the only elements are the partial derivatives of the model equations $f\left(x_{k}, u_{k}\right)$ and $h\left(x_{k}, u_{k}\right)$ both with respect to the states $x$ and the input $u$. These well structured Jacobians also result in a favourable, sparse, structure of the KKT matrix. As an example, the inversion of a $2^{\text {nd }}$ order model and a given output of 500 samples results in a KKT matrix with less than $1 \%$ non zero elements.

The constrained Gauss-Newton algorithm can be summarised as follows:

1) The variable vector $w$ is initialised, for example by setting $w^{0}=0$.

2) The KKT system is constructed based on the available model $\hat{P}$ and the partial derivatives of the model equations to the input and states. 
3) The solution of the KKT equation (9) determines an optimal search direction $\Delta w^{*}$, which is used to iterate on the variable vector, using: $w^{i+1}=w^{i}+\Delta w^{*}$.

4) After a stopping criterion has been met, the final variable vector $w$ contains the states $x$ and the input $u$ associated with the given output $y^{*}$.

\section{Simulation AND EXPERIMENTAL RESULTS}

This section discusses the validation of the algorithms that are presented in sections II and III, both via simulations and experiments. Section IV-A describes the nonlinear model structure that is used. The validation of the presented nonlinear model inversion algorithm is done numerically, i.e. via simulations. A comparison between the linear ILC approach (1) and the nonlinear ILC approach (3) is also done numerically. The nonlinear ILC is validated experimentally on an electric circuit called the Silverbox, described in section IV-B. The numerical validation of the model inversion algorithm and of the linear and nonlinear ILC are treated in sections IV-C and IV-D respectively. Section IV-E discusses the experimental validation of the nonlinear ILC algorithm.

\section{A. Polynomial nonlinear state-space model}

The discrete-time polynomial nonlinear state-space (PNLSS) model, described in detail in [10], has the following form:

$$
\begin{aligned}
x_{k+1} & =A x_{k}+B u_{k}+E \zeta\left(x_{k}, u_{k}\right) \\
y_{k} & =C x_{k}+D u_{k}+F \eta\left(x_{k}, u_{k}\right) .
\end{aligned}
$$

In this model structure $x$ is the state vector of length $n$, with $n$ the order of the model, $u$ is the input vector, and $y$ is the output vector. Although the PNLSS model is defined for MIMO systems, only SISO systems are considered in this paper. The subscript $k$ denotes the $k^{\text {th }}$ time instant in the discrete sequence.

The PNLSS model consists of a linear part, augmented with the nonlinear terms $E \zeta$ and $F \eta$. The linear part is characterized by the matrices $A \in \mathbb{R}^{n \times n}, B \in \mathbb{R}^{n \times 1}, C \in \mathbb{R}^{1 \times n}$ and $D \in \mathbb{R}$. The vectors $\zeta \in \mathbb{R}^{n_{\zeta}}$ and $\eta \in \mathbb{R}^{n_{\eta}}$ contain monomials in $x_{k}$ and $u_{k}$; the matrices $E \in \mathbb{R}^{n \times n_{\zeta}}$ and $F \in \mathbb{R}^{1 \times n_{\eta}}$ contain the coefficients associated with those monomials, with $n_{\zeta}$ and $n_{\eta}$ the number of monomials in $\zeta$ and $\eta$ respectively.

\section{B. The Silverbox system}

The system that is considered for the numerical and experimental validation of the proposed algorithms is an electric circuit, called the Silverbox, developed at Vrije Universiteit Brussel [11]. The structure of this circuit is equivalent to a mass-spring-damper system with a cubically proportional spring.

Three models are used to describe this system. The most accurate model is a $2^{\text {nd }}$ order PNLSS model with both $2^{\text {nd }}$ and $3^{\text {rd }}$ degree nonlinear terms, and will be called $\hat{P}_{1}$. It is used for the numerical validation of the model inversion algorithm,

\begin{tabular}{|c|c|c|}
\hline Name & Type & Use \\
\hline$\hat{P}_{1}$ & PNLSS, $2^{\text {nd }}+3^{\text {rd }}$ degree NL & Exp. validation of NILC \\
\hline$\hat{P}_{2}$ & PNLSS, $3^{\text {rd }}$ degree NL & Num. validation of NILC \\
\hline$\hat{P}_{3}$ & Linear & Num. validation of LILC \\
\hline
\end{tabular}

TABLE I

THREE MODELS USED IN NUMERICAL AND EXPERIMENTAL VALIDATION
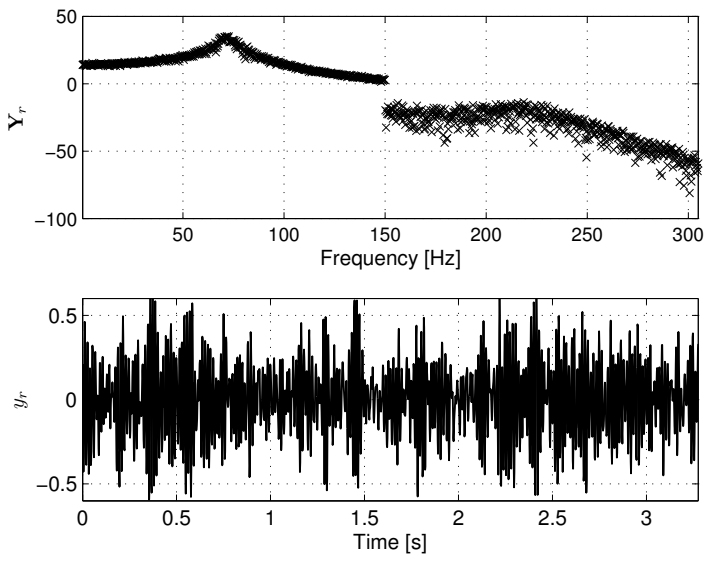

Fig. 2. Reference signal in the frequency domain (top) and time domain (bottom)

described in section IV-C, and for the experimental validation of the nonlinear ILC algorithm, described in section IV-E. It also serves as a sytem for the simulation of the linear and nonlinear ILC algorithms, in section IV-D. Another, less accurate, $2^{\text {nd }}$ order PNLSS model with only $3^{\text {rd }}$ degree nonlinear terms is used in the simulation of the nonlinear ILC algorithm, to introduce model inaccuracies. This model will be called $\hat{P}_{2}$. [10] and [12] discuss the identification of these models in detail. The third model is a linear, non-parametric model, used in the simulation of the linear ILC algorithm. It consists of a simulated FRF of the model $\hat{P}_{1}$. It is used during all iterations of the linear ILC algorithm. This model will be called $\hat{P}_{3}$. The three models are summarised in table I.

Two different reference signals are used during simulation and experiments. The first reference signal is used for the numerical validation of the model inversion algorithm and for the experimental validation of the nonlinear ILC algorithm, and is shown in figure 2. It is obtained by exciting the electric circuit with a random phase multisine, of 2000 samples, bandlimited to $150 \mathrm{~Hz}$, with an RMS value of $80 \mathrm{mV}$, and measuring the output. The nonlinear behavior of the system can be observed in the output spectrum above the bandwidth of the excitation signal $(150 \mathrm{~Hz})$. The reference signal is therefore not bandlimited, but it is the response of the system to a bandlimited input. The second reference signal, used for the simulation of the linear and nonlinear ILC algorithms, is obtained in a similar way but using model $\hat{P}_{1}$ as the system. Also the RMS value of the input is lowered to 28 $\mathrm{mV}$. This is necessary to allow a comparison of the linear and 

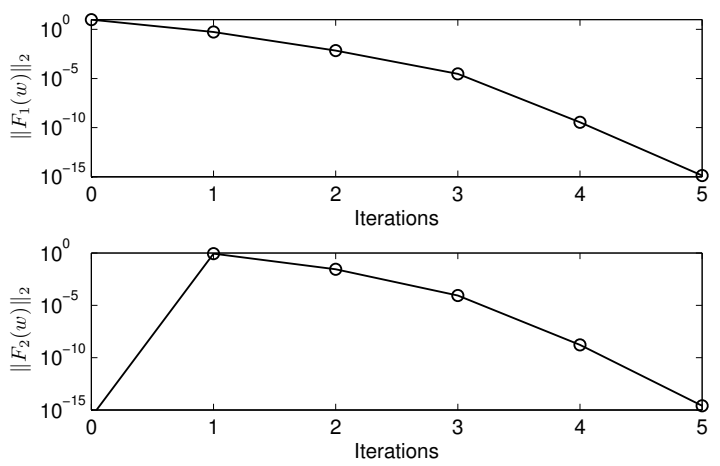

Fig. 3. Monotone convergence of the objective (top) and constraint (bottom) function norms

nonlinear ILC algorithms, since the linear ILC algorithm does not converge for stronger input signals, due to the stronger nonlinear contributions to the reference signal.

\section{Numerical validation of model inversion}

Model $\hat{P}_{1}$ and the reference signal shown in figure 2 are used to test the model inversion algorithm introduced in chapter III. For the initial estimate of the variable vector, both the states $x$ and the input $u$ are set to zero.

Figure 3 shows the evolution of the norm of the objective function, $\left\|F_{1}(w)\right\|_{2}$, and constraint function, $\left\|F_{2}(w)\right\|_{2}$, over the iterations of the algorithm. From this figure it is clear that the algorithm shows a monotone convergence towards machine precision for both functions, after the first iteration. The constraint function is zero for the initial estimate $w=0$, but subsequent values of the variable vector $w$ violate the constraint $F_{2}(w)=0$ in order to guarantee a decrease in the objective function. However, after the first iteration the constraint function also shows monotone convergence towards machine precision.

The used reference signal contains 2000 samples. Since the variable vector $w$ of the dynamic optimization problem consists of the two state variables and an input for each considered time step, $w$ contains 6000 elements. Despite the many variables, the constrained Gauss-Newton algorithm converges in less than 6 seconds on a $2.00 \mathrm{GHz}$ dual core PC with 2 GB RAM. With efficient memory usage it is possible to increase the length of the reference signal to about 7000 samples, or 21000 optimization variables. The model inversion method discussed in [1] is limited to about 500 optimization variables. This increase in efficiency of the constrained Gauss-Newton algorithm is the result of the structure and sparsity of the dynamic optimization problem, that is exploited during the solution of (9) in the CGN algorithm. Furthermore, the present formulation of the problem in time domain allows for the model inversion to be split into multiple subsequent inversion problems. The consistency of the calculated signals should then be enforced by adding appropriate constraints at the split point. This is a topic for further research.
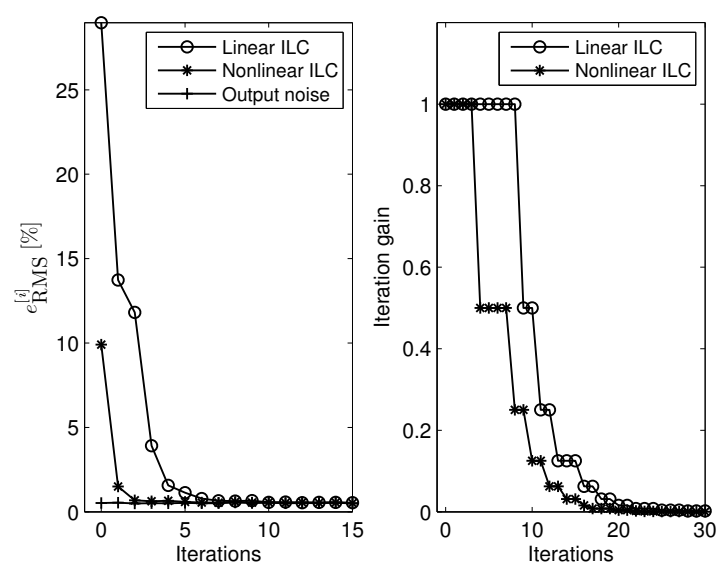

Fig. 4. Comparison of linear and nonlinear ILC in simulation: convergence of the relative tracking error (left), and reduction of the iteration gain (right)

\section{Numerical validation of linear and nonlinear ILC}

This section compares the performance of the nonlinear ILC algorithm (3) with the linear ILC algorithm (1) for the considered nonlinear system. The system is represented in this numerical validation by the model $\hat{P}_{1}$. The second, smaller reference signal is used in order to allow convergence of the linear ILC algorithm.

The nonlinear ILC algorithm uses model $\hat{P}_{2}$ to describe the system, while the linear ILC algorithm uses the linear model $\hat{P}_{3}$. Normally distributed random noise with an RMS value of $0.4 \mathrm{mV}$ is added to the output to simulate measurement noise. This noise level is similar to the measurement noise that is observed during the experimental validation of the nonlinear ILC algorithm. For both algorithms, the iteration gain in (1) and (3) is controlled using the second approach, described in section II.

Figure 4 shows the results of 30 iterations of both ILC algorithms. It is clear from this figure that, while both algorithms converge due to the reduced nonlinearity of the system, the nonlinear ILC algorithm converges faster than the linear ILC algorithm. The initial relative tracking error is lower for the nonlinear approach, because the PNLSS model $\hat{P}_{2}$ is more accurate than model $\hat{P}_{3}$ used in the linear approach. The convergence of both algorithms is clear from the evolution of the iteration gain, shown in figure 4 on the right. When the tracking error approaches the noise level, the gain is automatically reduced until the input signal is no longer updated, indicating convergence. The reduction of the iteration gain starts sooner for the nonlinear ILC algorithm.

\section{E. Experimental validation of nonlinear ILC}

This section discusses the experimental validation of the nonlinear ILC on the Silverbox. Reference signal 1, shown in figure 2, is used such that the nonlinearities are sufficiently excited. The nonlinear ILC algorithm uses model $\hat{P}_{1}$ to describe the system, in other words the most accurate PNLSS model available. The initial input signal that is applied to the system is the solution of the model inversion algorithm 

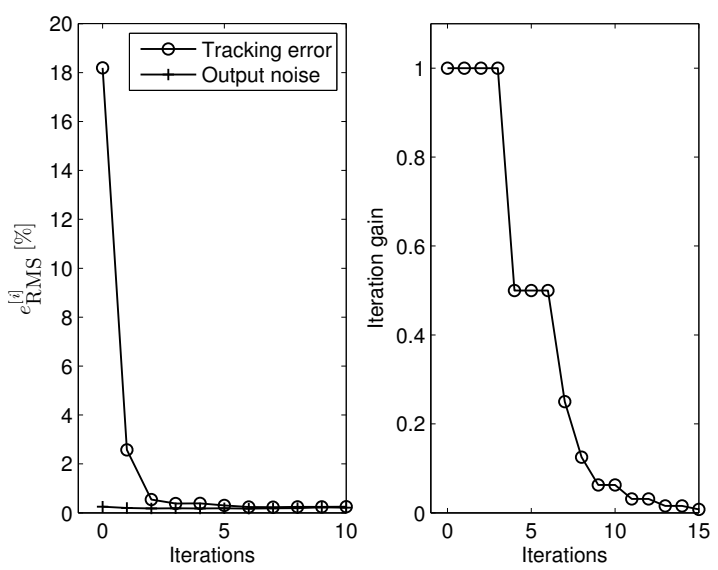

Fig. 5. Experimental validation of nonlinear ILC: convergence of the relative tracking error (left), and reduction of the iteration gain (right)

applied to the reference signal.

Figure 5 shows the evolution of the relative tracking error over the iterations of the nonlinear ILC algorithm. The initial input signal results in a relative tracking error of about 18 percent. This is due to the limited accuracy of the model and due to measurement noise. After about 10 iterations the relative tracking error reaches the level of the measurement noise. The convergence of the algorithm is also clear from the evolution of the iteration gain, shown in figure 5 on the right. After about 15 iterations there is no update of the control signal anymore, indicating convergence. Figure 6 shows the frequency spectra of the reference signal, the measurement noise, the initial tracking error and the tracking error after 10 iterations of the ILC. The spectrum of the measurement noise is estimated by measuring multiple periods of the output signal. It is clear from this figure that the tracking error is succesfully reduced to the level of the measurement noise. Figure 6 also shows the tracking results in the time domain. It is clear that a very accurate tracking control is obtained after convergence of the ILC algorithm.

\section{CONCLUSIONS}

This paper discusses iterative learning control (ILC) for nonlinear systems. The crucial part of model based nonlinear ILC is the inversion of a nonlinear model, i.e. the calculation of the input signal corresponding to a given output signal. This problem is formulated as a dynamic optimization problem in time domain, which can be solved efficiently using a constrained Gauss-Newton (CGN) algorithm. The present formulation introduces structure and sparsity to the optimization problem, which is exploited by the CGN algorithm.

The model inversion algorithm is numerically validated in simulations. The nonlinear ILC using this model inversion approach is compared to a standard model based linear ILC method in simulations.

The nonlinear ILC is also validated experimentally on an electric circuit, described by a polynomial nonlinear state space model. With increased nonlinear behaviour of the
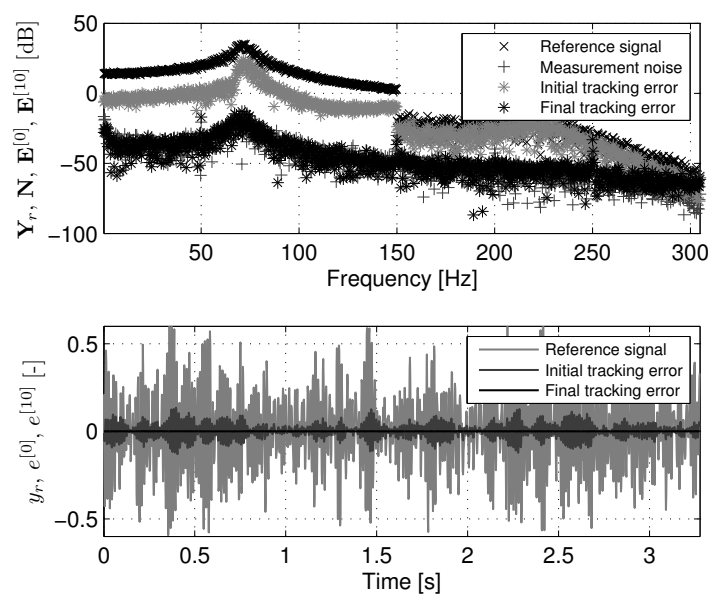

Fig. 6. Experimental validation of nonlinear ILC: frequency spectra of the reference signal $(\mathrm{x})$, the measurement noise $(+)$, the initial tracking error $(*)$ and the tracking error after 10 iterations (*) (top), and the reference signal, initial and final tracking error in time domain (bottom)

system, for which linear ILC fails to converge, the nonlinear ILC shows fast convergence and accurate tracking control.

Further research will focus on the nonlinear learning law, and on the possibility to increase the length of the reference signal by splitting the model inversion problem into multiple sub-problems, using constraints to guarantee the consistency of the calculated feedforward signals.

\section{REFERENCES}

[1] K. Smolders, M. Volckaert, J. Swevers, Tracking control of nonlinear lumped mechanical continuous-time systems: a model-based iterative learning approach, Mechanical Systems and Signal Processing 22 (2008) 1896-1916.

[2] D. Bristow, M. Tharayil, A. Alleyne, A survey of iterative learning control, Control Systems Magazine, IEEE 26 (3) (2006) 96-114.

[3] G. B. Giannakis, E. Serpedin, A bibliography on nonlinear system identification, Signal Processing 81 (2001) 533-580.

[4] J. D. Cuyper, M. Verhaegen, J. Swevers, Off-line feed-forward and feedback control on a vibration rig, Control Engineering Practice 11 (2) (2003) $129-140$.

[5] E. Baake, M. Baake, H. Bock, K. Briggs, Fitting ordinary differential equations to chaotic data, Phys. Rev. A 45 (1992) 5524-5529.

[6] H. Bock, Recent advances in parameter identification techniques for ODE, in: P. Deuflhard, E. Hairer (Eds.), Numerical Treatment of Inverse Problems in Differential and Integral Equations, Birkhäuser, Boston, 1983.

[7] H. Bock, Randwertproblemmethoden zur Parameteridentifizierung in Systemen nichtlinearer Differentialgleichungen, Vol. 183 of Bonner Mathematische Schriften, Universität Bonn, Bonn, 1987.

[8] H. Bock, E. Eich, J. Schlöder, Numerical Solution of Constrained Least Squares Boundary Value Problems in Differential-Algebraic Equations, in: K. Strehmel (Ed.), Numerical Treatment of Differential Equations, Teubner, Leipzig, 1988.

[9] J. Nocedal, S. J. Wright, Numerical Optimization, 2nd Edition, Springer Series in Operations Research and Financial Engineering, Springer, 2006.

[10] A. Van Mulders, J. Schoukens, M. Volckaert, M. Diehl, Two nonlinear optimization methods for black box identification compared, submitted to the 15th IFAC Symposium on System Identification (SYSID 2009).

[11] R. Pintelon, J. Schoukens, System Identification: A Frequency Domain Approach, Wiley-IEEE Press, 2001.

[12] J. Paduart, J. Schoukens, R. Pintelon, T. Coen, Nonlinear state space modelling of multivariable systems, $14^{\text {th }}$ IFAC Symposium on System Identification (SYSID 2006) (2006) 565-569. 